\title{
三维多孔石墨烯/铂钯双金属杂化体作为高性能的甲醇氧化电催化剂
}

\author{
孙红梅 ${ }^{a, b} \quad$ 曹林园 ${ }^{a}$ 逯乐慧 $*, a$ \\ ( ${ }^{a}$ 中国科学院长春应用化学研究所 电分析化学国家重点实验室 长春 130022) \\ ${ }^{b}$ 中国科学院大学 北京 100039)
}

\begin{abstract}
摘要 通过简便的溶剂热还原方法以及冰模板自组装技术, 成功构建了三维多孔石墨烯/铂钯双金属杂化体. 三维多孔 的结构以及可调的组成成分赋予这种杂化体大的比表面积和高的催化活性, 使其展现了较高的催化甲醇氧化的能力, 为构筑新型高效的甲醇燃料电池催化剂提供了一个新的平台.
\end{abstract}

关键词 石墨烯; 双金属; 燃料电池; 冰模板; 溶剂热

\section{Three Dimensional Porous Graphene/PtPd Bimetallic Hybrids as High-performance Electrocatalyst for Methanol Oxidation}

\author{
Sun, Hongmei ${ }^{a, b}$ Cao, Linyuan ${ }^{a} \quad$ Lu, Lehui*, $^{*}$ \\ ( ${ }^{a}$ State Key Laboratory of Electroanalytical Chemistry, Changchun Institute of Applied Chemistry, \\ Chinese Academy of Sciences, Changchun 130022) \\ ( ${ }^{b}$ Graduate University of the Chinese Academy of Sciences, Beijing 100039)
}

\begin{abstract}
The development of fuel cells is highly dependent on the exploration of the efficient electrocatalyst. However, up to now, constructing high-quality hybrids with large electrochemical surface area (ECSA) through a facile method has remained a great challenge. In this paper, a novel approach for producing three dimensional porous graphene/PtPd bimetallic hybrids was developed by combining the solvothermal strategy with the ice template technique. First, a simple solvothermal route were employed for preparing PtPd bimetallic nanoparticle supported on graphene (PPG) hybrids by simultaneously forming bimetallic nanoparticles and reducing graphene oxide (GO). Then, three dimensional porous graphene/PtPd bimetallic hybrids are obtained via the ice templation of an aqueous suspension comprised of the PPG and phthalic acid diethylene glycol diacrylate (PDDA). The as-prepared 3D PPG were characterized by transmission electron microscopy (TEM), high-resolution TEM (HRTEM), X-ray diffraction (XRD), scanning electron microscopy (SEM), X-ray photoelectron spectroscopy (XPS), Raman spectroscopy and electrochemical technique. It is interesting to find that the loading of PtPd bimetallic nanoparticles on the surface of graphene could be controlled by simply changing the initial weight ratio of the precursors. Furthermore, the hydrophilicability of PDDA plays an important role on the fabrication of 3D porous graphene/PtPd bimetallic hybrids. Most importantly, this special morphology endows the 3D PPG hybrids with larger ECSA and more catalytic sites compared with the PPG and commercial E-TEK Pt/C catalysts, and thus leads to much higher catalytic activity towards methanol oxidation reaction. The details are shown as follows. (a) The ECSA value of the as-prepared 3D PPG hybrids is tested to be $98.7 \mathrm{~m}^{2} \cdot \mathrm{g}^{-1}$, while the ECSA values of PPG and E-TEK Pt/C catalysts are tested to be 61.3 and $46.5 \mathrm{~m}^{2} \cdot \mathrm{g}^{-1}$, respectively. (b) The mass current density for methanol oxidation in 3D PPG hybrids is higher than those of PPG and E-TEK $\mathrm{Pt} / \mathrm{C}$ catalysts and the corresponding potential on 3D PPG hybrids is much lower than that on PPG and E-TEK Pt/C catalysts at a given oxidation current density. (c) The as-prepared 3D PPG hybrids catalyst exhibits greater poisoning tolerance than the PPG and E-TEK Pt/C catalysts during methanol oxidation. All results reveal that these 3D PPG hybrids can provide a new and versatile platform for the development of high-performance electrocatalyst for methanol oxidation.

Keywords graphene; bimetal; fuel cell; ice template; solvothermal
\end{abstract}

\section{1 引言}

目前, 全球能源危机迫切需要人们不断地开发新的 储能装置. 甲醇燃料电池由于其能量密度高、易操作等 优势, 被认为是最有前途的清洁能源器件之一[1 3]. 研 究表明, 燃料电池的性能在很大程度上依赖于所用催化
剂的性能 ${ }^{[4 \sim 8]}$. 因此，寻找高效的催化剂对于探索甲醇 氧化反应显得十分必要.

铂是目前发现的最有效的甲醇氧化催化剂之一. 不 同形状的铂纳米粒子，比如，立方块铂 ${ }^{[4]}$ 二二十四面体 铂 ${ }^{[5]}$,介孔铂 ${ }^{[6,7]}$, 以及枝状体铂均被报道可作为高效的 甲醇氧化催化剂 ${ }^{[8]}$. 虽然基于铂的甲醇燃料电池催化剂

* E-mail: lehuilu@ciac.jl.cn; Tel.: 0431-85262418; Fax: 0431-85062406

Received November 15, 2012; published March 1, 2013.

Supporting information for this article is available free of charge via the Internet at http://sioc-journal.cn.

Project supported by the National Basic Research Program of China (973 Program; No. 2010CB933600).

项目受国家基础研究 973 (No. 2010CB933600)资助. 
的研究已经取得了显著的进展, 然而, 一些挑战性的问 题，例如，低的甲醇转换率以及缓慢的甲醇电氧化动力 学问题仍然是关系到甲醇然料电池商品化的重要技术 障碍 ${ }^{[9,10]}$.

为了克服这一难题, 以及降低贵金属铂的使用量进 而降低催化剂成本, 将高活性铂纳米结构固载到表面积 高、导电性好和低成本的载体纳米材料上显得十分必 要 ${ }^{[11 ~ 16]}$. 由于具有高的导电性 $\left(103 \sim 104 \mathrm{~S} \cdot \mathrm{m}^{-1}\right)$, 大的 比表面积(计算值, $2630 \mathrm{~m}^{2} \cdot \mathrm{g}^{-1}$ ), 独特的石墨化基面结 构和潜在的低成本, 石墨烯纳米片在构建新的杂化催化 剂方面展现了巨大的优势 ${ }^{[9,10]}$. 已有文献报道通过电沉 积，自组装，共还原等方法来实现石墨烯/金属纳米 星 ${ }^{[17]}$, 石墨烯/金属纳米粒子 ${ }^{[9]}$, 石墨烯/金属纳米片 ${ }^{[10]}$ 等材料的制备. 另一方面, 由于具有比单一组成的金属 纳米粒子更强的催化性能, 双金属纳米粒子已经受到了 越来越多的关注 ${ }^{[18 ~ 21]}$. 比如, 金/铂双金属纳米粒子能 大大地增加铂的表面积, 这对于提高铂的催化活性十分 必要 ${ }^{[18 ~ 20]}$. 最近, Guo 等结合石墨烯和双金属的优势, 制备了高质量的石墨烯/铂钯双金属纳米枝状物杂化体, 并对其甲醇氧化催化性能进行了研究 ${ }^{[13]}$. 该复合材料 显示了较高的甲醇氧化催化活性, 然而, 一些潜在的影 响催化剂性能的因素: 如纳米粒子容易聚集, 在石墨烯 表面的负载量较低, 依然存在, 亟待解决 ${ }^{[22,23]}$.

三维组装纳米结构由于能在较小的范围内提供较 多的吸附位点, 保证快速的分子传输, 因此能较好地解 决上述问题 ${ }^{[24]}$. 近期, Giannelis 及其合作者利用冰模板 技术构筑了石墨烯/铂纳米粒子三维组装结构. 该材料 对甲醇氧化反应具有良好的效果 ${ }^{[24]}$. 然而, 迄今为止, 有关三维多孔石墨烯/双金属纳米材料的组装仍未有报 道. 其主要原因在于很难找到一种简便易行的方法来实 现三维多孔石墨烯/双金属杂化体的有效构筑.

基于以上考虑, 本文首次报道了一种构建三维多孔 铂钯双金属纳米粒子覆盖的石墨烯杂化材料(3D PPG) 的新方法. 与以往的报道相比, 该方法具有以下优势. 首先, 它只需将前驱物分子进行一步溶剂热反应, 便可 得到石墨烯/铂钯双金属(PPG)杂化体. 这为材料的商品 化生产提供了前提. 其次, 该多孔石墨烯/铂钯双金属杂 化体只需简单地与邻苯二甲酸二乙二醇二丙烯酸酯 (PDDA)的溶液混合、冷冻、干燥处理, 即可得到稳定的 三维多孔杂化体. 这避免了使用传统的方法(模板构筑、 模板刻蚀等)来制备三维多孔材料，大大简化了制备路 线. 第三, 在制备过程中所使用的试剂均为廉价易得的 试剂, 从而使得该方法为一个低成本的合成方法. 更为 重要的是, 该材料用作电化学传感平台时, 展现了较高 的吸氢/脱氢峰和金属氧化物的形成/还原峰, 以及较高 的甲醇氧化峰电流. 这说明了所制备的 3D PPG 杂化体 在燃料电池领域具有巨大的应用潜力.

\section{2 结果与讨论}

\subsection{PPG 的制备及性质表征}

溶剂热是一个很有效的制备纳米材料的方法. 相比 于静电组装，它具有反应速率快和反应产物均一的优 点 ${ }^{[25]}$. PPG 可以通过在 $180{ }^{\circ} \mathrm{C}$ 条件下, 将含有 $\mathrm{GO}, \mathrm{Pt}$, $\mathrm{Pd}$ 前躯体和聚丙烯酸(PAA)的乙二醇 $(\mathrm{EG})$ 溶液在反应 釜中进行反应得到. 在这里, EG 同时起到了溶剂与还原 剂的作用. 低倍率的透射电镜图片显示, 在石墨烯的表 面分散了许多尺寸较小、粒径均一的纳米粒子, 并且很 难观察到散落的纳米粒子(图 1). 这说明了纳米粒子与 石墨烯之间具有较强的作用力. 放大的透射电镜图片 (图 1c)显示了这些纳米粒子的尺寸范围在 3.5 4.8 nm 之间，平均粒径为 $4.2 \mathrm{~nm}$. 而高分辨透射电镜分析则揭 示出纳米粒子的晶格间距为 $2.3 \AA$, 对应面心立方的 (111)面. 以上结果表明我们已成功得到了 PPG 杂化体.
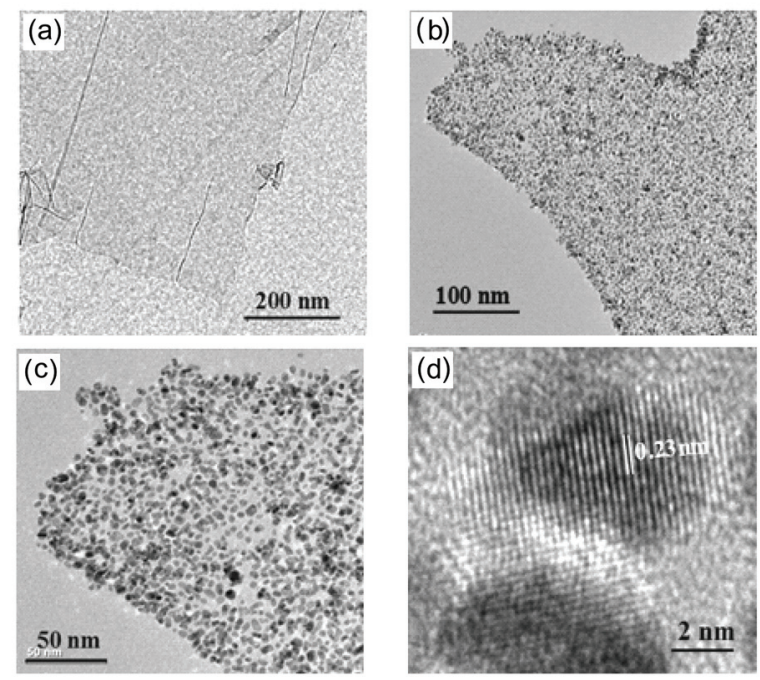

图 $1 \mathrm{GO}(\mathrm{a})$ 和 $\mathrm{PPG}$ 材料 $(\mathrm{b} \sim \mathrm{d})$ 的透射电镜图和(d)高分辨透射电镜表 征

Figure 1 TEM $(\mathrm{a} \sim \mathrm{c})$ and HRTEM (d) images of (a) GO and $(\mathrm{b} \sim \mathrm{d})$ PPG

为确认 PPG 纳米材料的化学结构, 我们对该材料 进行了 XRD 表征. 如图 2 所示, $2 \theta=39.89^{\circ}, 46.52^{\circ}$, $67.92^{\circ}, 81.82^{\circ}$ 和 $86.22^{\circ}$ 的谱峰分别归属于立方晶体结构 PtPd 的(111), (200), (220), (311)和(222)面衍射峰 ${ }^{[26]}$. 而 $26^{\circ}$ 附近的衍射峰可以归属为石墨烯中石墨化碳的(002) 面. 此外, 根据 Scherrer 公式, 由(111)衍射峰计算得到 的金属纳米粒子的平均粒径为 $4.2 \mathrm{~nm}$, 这与 TEM 结果 一致.

接下来，我们进一步采用 XPS 对 GO 和制备的 PPG 进行了元素成分分析(图 3). 结果显示, GO 中存在四种 碳键: C-C (284.6 eV), C-O (286.7 eV), C=O (287.8 $\mathrm{eV})$, 以及 $\mathrm{O}-\mathrm{C}=\mathrm{O}(288.7 \mathrm{eV})$. 当形成 $\mathrm{PPG}$ 后, 其 $\mathrm{XPS}$ 图谱发生了明显的变化. 具体表现为, 与碳氧键相关的 峰强度大大减弱, 而与 $\mathrm{C}-\mathrm{C}$ 或 $\mathrm{C}-\mathrm{H}(284.6 \mathrm{eV})$ 相关的 


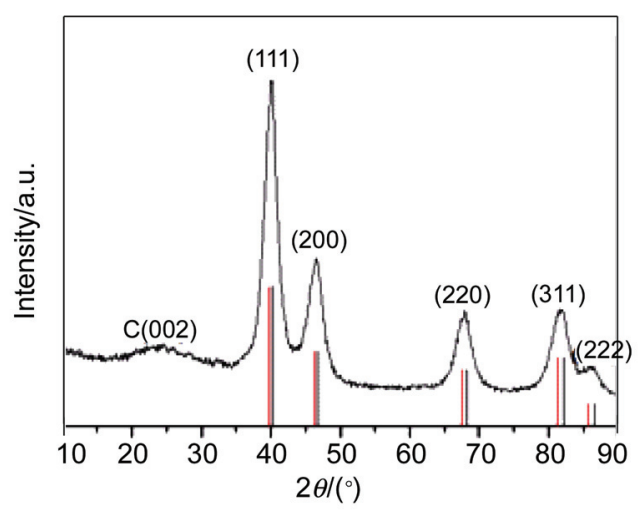

图 2 PPG 材料的 XRD 表征

Figure 2 XRD pattern of PPG
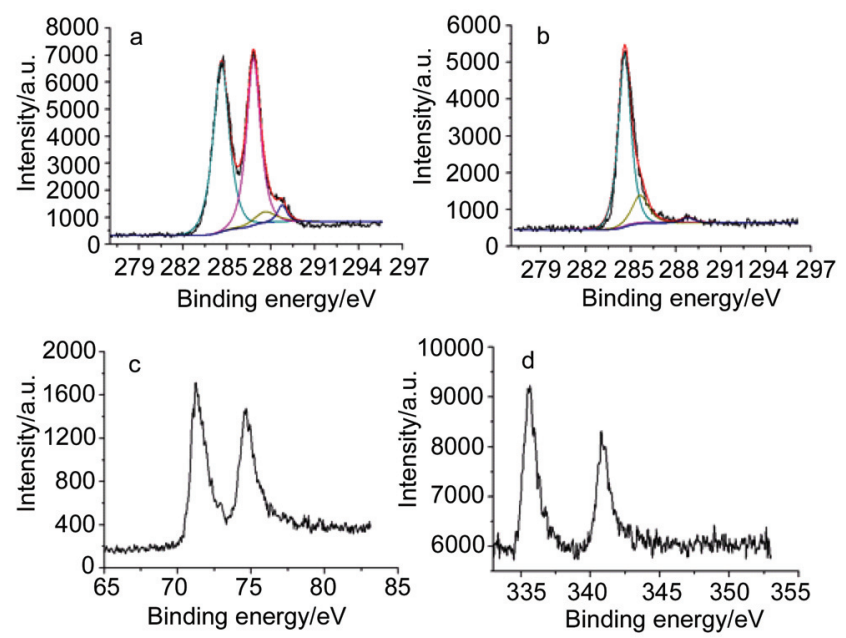

图 $3 \mathrm{GO}(\mathrm{a})$ 和 PPG $(\mathrm{b} \sim \mathrm{d})$ 材料的 XPS 表征

Figure 3 XPS patterns of GO (a) and PPG $(b \sim d)$

a, b: C1s; c: Pt4f, d: Pd4f

峰占据主导地位(图 3c, d). 这些结果表明 GO 已经被还 原为石墨烯. 除此之外, PPG 的 XPS 图谱还显示出铂的 $\mathrm{Pt} 4 \mathrm{f}$ 峰和钯的 Pd $4 \mathrm{f}$ 峰. 其中, Pt4f $\mathrm{f}_{7 / 2}$ 和 Pt $4 \mathrm{f}_{5 / 2}$ 的结合能分 别位于 71.2 和 $74.8 \mathrm{eV}$. 这是典型的零价态铂的数值. 而 $\mathrm{Pd} 4 \mathrm{f}_{7 / 2}$ 和 $\mathrm{Pd} 4 \mathrm{f}_{5 / 2}$ 的结合能则分别位于 335.7 和 $341.8 \mathrm{eV}$, 这与文献报道的零价态的金属钯一致 ${ }^{[13]}$. 以上结果进 一步说明铂钯双金属纳米粒子被有效地固定在了石墨 烯的表面.

有趣的是，石墨烯表面金属纳米粒子的分布密度可 以通过简单地改变反应参数来调控. 例如, 当金属前躯 体的用量为 $0.01 \mathrm{mmol} / \mathrm{L}$ 时, 石墨烯表面的金属纳米粒 子的密度会显著降低; 当金属前躯体的量为 $0.1 \mathrm{mmol} / \mathrm{L}$ 时, 可以得到高粒子密度的 PPG. 然而, 粒子密度过高 将会导致纳米粒子的轻度聚集, 进而降低 PPG 在水中 的分散性(图 4).

值得注意的是, 当把 PPG 水溶液进行超声处理 $3 \mathrm{~h}$ 后, 金属纳米粒子依然能牢固地固定在石墨烯表面. 这 表明金属纳米粒子并不是简单地吸附在石墨烯表面 ${ }^{[10]}$. 此外, 所制备的 PPG 材料在水中还显示出了很好的分
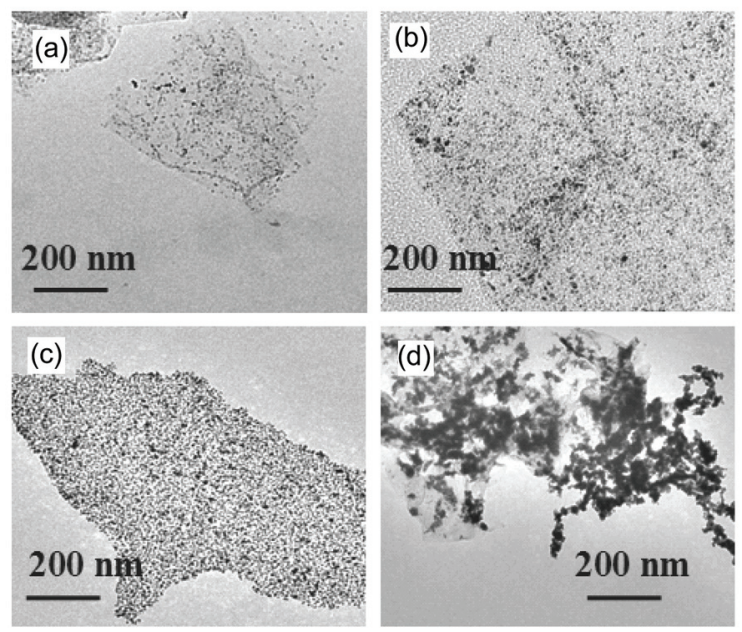

图 4 不同用量的金属前躯体所制备的 PPG 的透射电镜图. 金属前躯 体 $(\mathrm{Pt} / \mathrm{Pd}$ : $1 / 1)$ 的用量为 a: $0.01 \mathrm{mmol} / \mathrm{L} ; \mathrm{b}: 0.03 \mathrm{mmol} / \mathrm{L} ; \mathrm{c}: 0.05 \mathrm{mmol} / \mathrm{L}$; d: $0.1 \mathrm{mmol} / \mathrm{L}$

Figure 4 TEM images of the synthesized PPG with different initial amount of the precursors (Pt/Pd: 1/1). a: $0.01 \mathrm{mmol} / \mathrm{L}$; b: $0.03 \mathrm{mmol} / \mathrm{L}$; c: $0.05 \mathrm{mmol} / \mathrm{L}$; d: $0.1 \mathrm{mmol} / \mathrm{L}$

散性，这可能归因于在制备过程使用到了 PAA. 为了验 证这个猜想，我们用相同的方法，但不加入 PAA 来制备 PPG 材料. 结果在石墨烯表面只得到了聚集的散乱分布 的纳米粒子(图 5a). 这表明 PAA 在控制金属纳米粒子的 尺寸和分布过程中起到了重要作用. 此外, 纳米粒子的 结构也会随溶剂组成的变化而变化. 当溶剂组成为 $\mathrm{EG}: \mathrm{H}_{2} \mathrm{O}=1: 1$ 时, 得到的纳米粒子为粒径较大的树 枝状纳米结构, 而非尺寸均一的粒子结构(图 5b). 其原 因尚在进一步研究中.
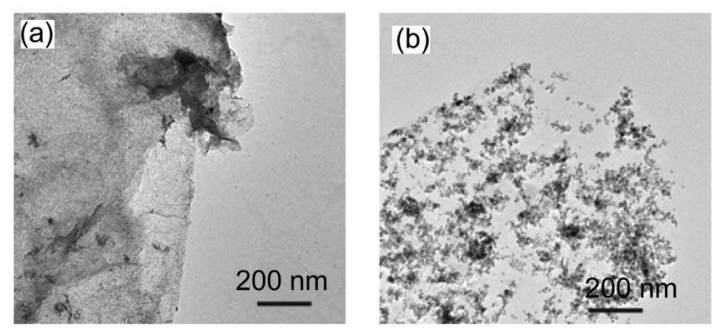

图 $5 \mathrm{PPG}$ 在改变实验条件下的 TEM 图, 图中标尺为 $200 \mathrm{~nm}$, 金属前 躯体 $(\mathrm{Pt} / \mathrm{Pd}: 1 / 1)$ 的用量为 $0.05 \mathrm{mmol} / \mathrm{L}$. a: 无 $\mathrm{PAA}$ 反应; b: $\mathrm{EG}: \mathrm{H}_{2} \mathrm{O}$ 为 $1: 1$

Figure 5 TEM images of PPG at different experimental conditions. The scale bar was $200 \mathrm{~nm}$ and the amount of the precursors was $(\mathrm{Pt} / \mathrm{Pd}=1 / 1)$ $0.05 \mathrm{mmol} / \mathrm{L}$. a: without PAA; b: $\mathrm{EG}: \mathrm{H}_{2} \mathrm{O}=1: 1$

Raman 光谱是一种强大的、无损坏的用来区分有 序和无序的碳晶体结构的手段 ${ }^{[28]}$. 石墨烯的 Raman 光 谱具有 $\mathrm{D}$ 带和 $\mathrm{G}$ 带. $\mathrm{D}$ 带中 $1348 \mathrm{~cm}^{-1}$ 附近的峰是由 $\mathrm{sp}^{3}$ 杂化的 $\mathrm{C}-\mathrm{C}$ 单键的伸缩振动产生的; $\mathrm{G}$ 带中 $1595 \mathrm{~cm}^{-1}$ 附近的峰则代表结晶石墨 $E_{2 \mathrm{~g}}$ 的震动模式. 图 6 为 $\mathrm{GO}$, $\mathrm{EG}$ 还原的 $\mathrm{GO}(\mathrm{rGO})$ 以及 $\mathrm{PPG}$ 的 Raman 光谱图. 在波 长范围为 $1000 \sim 2000 \mathrm{~cm}^{-1}$ 内, $\mathrm{GO}$ 除了出现变宽的 $\mathrm{G}$ 带 外, 还出现了 $\mathrm{D}$ 带. 这是由于经过剧烈氧化之后, 石墨 


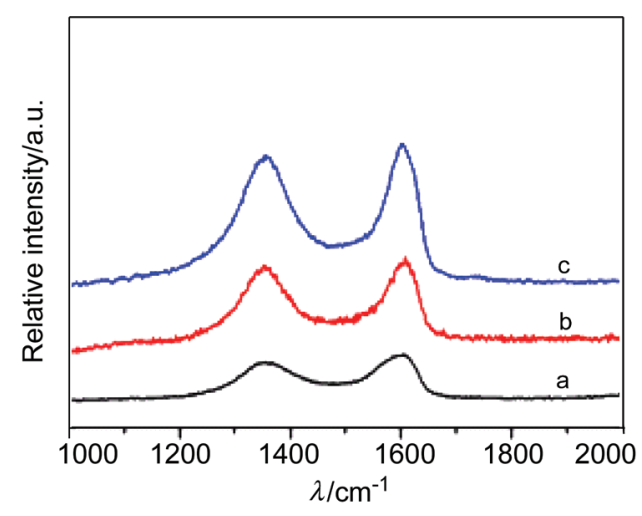

图 $6 \mathrm{GO}(\mathrm{a}), \mathrm{rGO}(\mathrm{b})$, 和 $\mathrm{PPG}(\mathrm{c})$ 的拉曼表征

Figure 6 Raman spectra of GO (a), rGO (b) 和 PPG (c)

烯平面内的 $\mathrm{sp}^{2}$ 区域逐渐变小而引起的. 当 $\mathrm{GO}$ 被 $\mathrm{EG}$ 还 原后, 拉曼谱图中仍含有 $\mathrm{D}$ 和 $\mathrm{G}$ 带. 但是, 与 $\mathrm{GO}$ 相比, $\mathrm{rGO}$ 具有较高的 $I_{\mathrm{D}} / I_{\mathrm{G}}$ 值. 这表明, 经 $\mathrm{EG}$ 还原后, 大量 的 $\mathrm{sp}^{3}$ 杂化的碳原子经脱氧后会重新形成 $\mathrm{sp}^{2}$ 杂化的碳 原子. 但可能由于重新形成的 $\mathrm{sp}^{2}$ 区域比氧化石墨烯的 要小, 使得还原后石墨烯的平均 $\mathrm{sp}^{2}$ 区域变小. 这反映 在拉曼谱图中即为 $I_{\mathrm{D}} / I_{\mathrm{G}}$ 的增大. 此外, 我们发现 $\mathrm{PPG}$ 的 $I_{\mathrm{D}} / I_{\mathrm{G}}$ 与 $\mathrm{rGO}$ 相比, 没有明显的改变. 这表明双金属纳米 粒子的功能化并没有改变石墨烯面内 $\mathrm{sp}^{2}$ 区域(决定石墨 烯的导电性)的大小.

\subsection{PPG 的三维结构组装}

鉴于三维结构的特殊性, 我们认为这种高密度的 PPG 被组装成三维结构后可能会在实际应用中发挥更 重大的作用. 为此, 我们采用一个简单的低温冷冻方法 来构建 3D PPG. 这个过程分两步进行: (1) 将 PPG 水溶 液与 PDDA 混合并滴涂在基底上; (2) 放入液氮中保持 2 $\mathrm{h}$, 然后置于 $25{ }^{\circ} \mathrm{C}$ 烘箱中干燥. 相应的扫描电镜图片如 图 7 所示. 片状的 PPG 相互交错, 构成多孔的三维网络 结构. 这些孔洞的形成与 PPG 和 PDDA 强的亲水性有 关. 由于亲水基团的存在, 使得在冷冻过程中, 水在这 些 PPG 片周围富集, 形成微核小液滴, 进而起到成核剂 的作用 ${ }^{[24]}$. 当冷冻干燥后, 水所占据的空间就变成孔的 结构. 由于 PPG 在水中的随机分布, 使得富集在其周围 的水形成无序的介孔和微孔, 最终组成一个相互连接的 多孔结构. 这可能会为电子的传输提供便利.
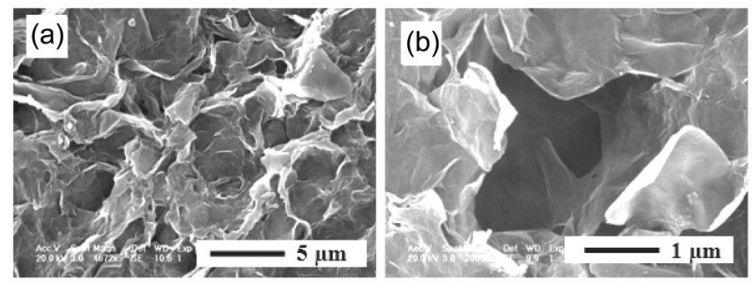

图 7 3D PPG 的扫描电镜图

Figure 7 SEM images of 3D PPG
接下来, 我们考察了 PDDA 的用量对于三维多孔杂 化体结构的影响. 如图 8 所示, 当改变 PPG 与 PDDA 的 质量比时, 所得到的杂化体结构将发生很大变化. 例如, 当不加入 PDDA 时, 所得到的杂化体虽然也能形成三维 结构, 但是 PPG 会发生一定程度的聚集, 从而形成无孔 的结构. 随着 PDDA 量的增加, 杂化体中的孔结构会慢 慢增多. 然而, 当 PDDA 的用量超过 $1 / 19$ 时, 所得到的 杂化体是一个接近致密的结构. 显然, 这对其电化学应 用是不利的. 因此, 经过优化, 我们确定 PGG 与 PDDA 质量比为 $1 / 19$ 作为制备 $3 \mathrm{D}$ PPG 的最佳比值.
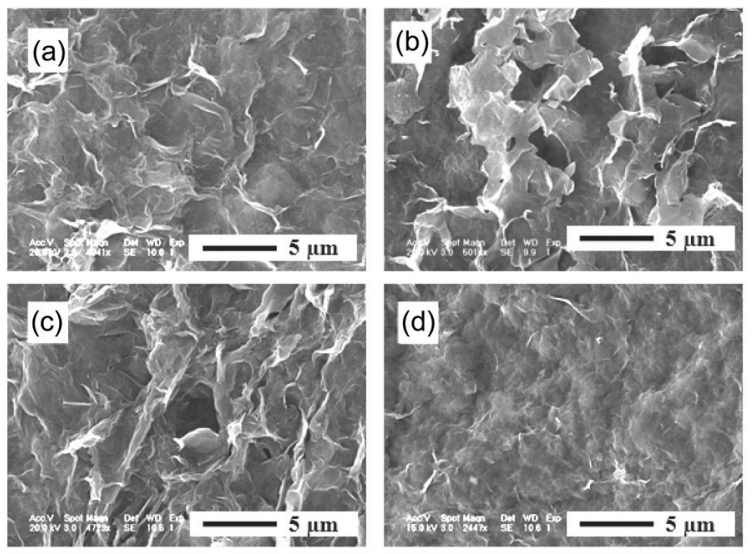

图 8 不同试验条件下的 3D PPG 的扫描电镜图. a: 无 PDDA; b: $\mathrm{PDDA} / \mathrm{PGG}$ 的质量比例为 $1 / 19 ; \mathrm{c}:$ PDDA/PGG 的质量比例为 $1 / 12 ; \mathrm{d}$ : PDDA/PGG 的质量比例为 $1 / 9$

Figure 8 SEM images of 3D PPG at different experimental conditions. a: without PDDA; b: PDDA/PGG $=1 / 19$ (the mass ratio); : PDDA/PGG $=1 / 12$ (the mass ratio); $\mathrm{d}: \mathrm{PDDA} / \mathrm{PGG}=1 / 9$ (the mass ratio)

\subsection{D PPG 电化学性质表征}

考虑到 3D PPG 结构的特殊性, 我们猜想它可能会 具有良好的催化性能. 因此, 我们用循环伏安法考察了 $3 \mathrm{D}$ PPG 的电化学属性. 通过用氢吸附-脱附峰 ${ }^{[29]}$, 测 量出了 3D PPG, PPG, E-TEK Pt/C 催化剂电化学活性面 积分别为 $98.7,61.3$ 和 $46.5 \mathrm{~m}^{2} \cdot \mathrm{g}^{-1}$ (图 9). 显然, 3D PPG 的催化面积远远高于 PPG, E-TEK Pt/C 商业催化剂, 以 及 Pt-Pd 双金属纳米枝状物 ${ }^{[13]}$ 等材料. 这很可能是因为 三维多孔结构具有较大的比表面积. 同时, 这也暗示了 3D PPG 具有较高的电化学活性, 可能是一个高效的催 化剂.

接下来，我们在酸性条件下考察了所制备的 3D PPG 催化甲醇氧化的性能. 图 10 比较了 3D PPG, PPG 以及商业 E-TEK C/Pt 催化剂在相同条件下催化甲醇氧 化的循环伏安图. 与商品化的 E-TEK 催化剂比较, PPG 在一个固定氧化电流密度处相应的电势更低. 这暗示了 PPG 材料具有较好的甲醇氧化催化性能. 而当 PPG 被构 筑成三维结构时, 其电势会进一步降低, 说明 3D PPG 材料更利于甲醇分子的氧化, 具有更强的催化性能. 这 种增强的催化活性很可能归属于以下主要因素: (1)石墨 


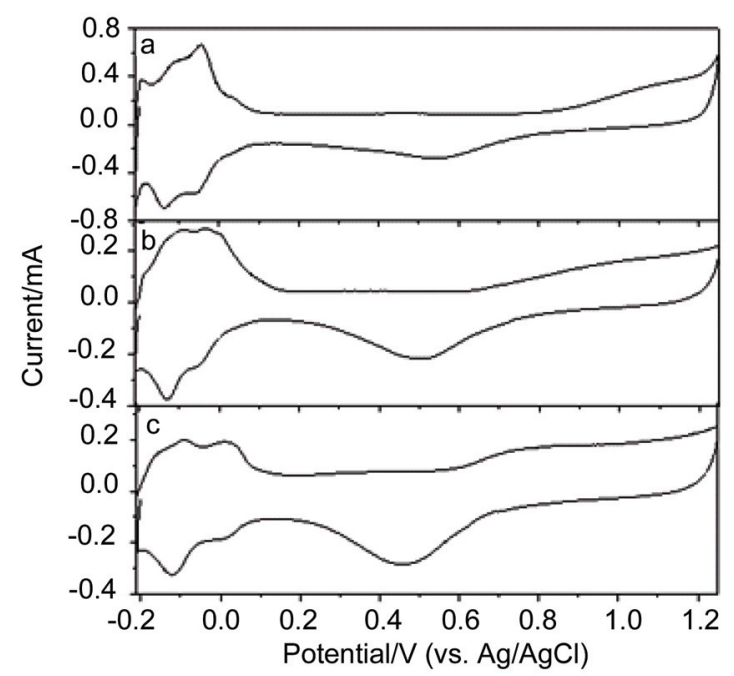

图 $93 \mathrm{D}$ PPG (a), PPG (b)和 E-TEK Pt/C 催化剂(c)修饰的玻碳电极在 氮气饱和的 $0.5 \mathrm{~mol} / \mathrm{L} \mathrm{H}_{2} \mathrm{SO}_{4}$ 中的循环伏安图. 扫速为 $50 \mathrm{mV} \cdot \mathrm{s}^{-1}$

Figure $9 \mathrm{CVs}$ of 3D PPG (trace a), PPG (b) (trace b), and E-TEK Pt/C (trace c) modified GC electrodes in a $\mathrm{N}_{2}$-sparged $0.5 \mathrm{~mol} / \mathrm{L} \mathrm{H}_{2} \mathrm{SO}_{4}$ solution at the scan rate of $50 \mathrm{mV} \cdot \mathrm{s}^{-1}$

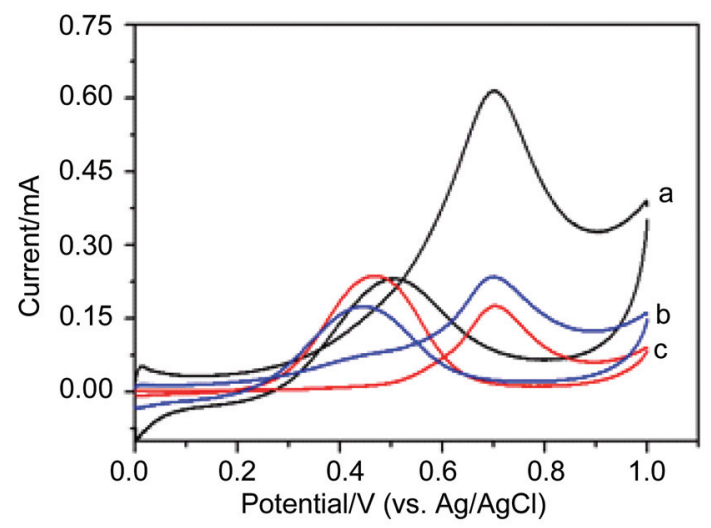

图 10 3D PPG (a), PPG (b)和 E-TEK Pt/C 催化剂(c)修饰的玻碳电极 在 $0.5 \mathrm{~mol} / \mathrm{L} \mathrm{H}_{2} \mathrm{SO}_{4}+1 \mathrm{~mol} / \mathrm{L} \mathrm{CH}_{3} \mathrm{OH}$ 溶液中的循环伏安曲线. 扫速为 $50 \mathrm{mV} \cdot \mathrm{s}^{-1}$

Figure $10 \mathrm{CVs}$ of 3D PPG (trace a), PPG (trace b), and E-TEK Pt/C (trace c) catalyst modified GC electrodes in a $0.5 \mathrm{~mol} / \mathrm{L} \mathrm{H}_{2} \mathrm{SO}_{4}$ solution containing $1 \mathrm{~mol} / \mathrm{L}$ methanol at the scan rate of $50 \mathrm{mV} \cdot \mathrm{s}^{-1}$

烯表面的双金属纳米粒子的高密度分布对于提高电化 学活性面积和催化活性非常有利; (2)石墨烯具有较高的 导电性，便于加快目标分子与电极间的电子传递速度； (3) 特殊的三维多孔结构能有效地加快分子在电极表面 的吸附脱附.

为了进一步提高 $3 \mathrm{D}$ PPG 的催化性能, 我们考察了 不同比例的铂钯对于甲醇催化性能的影响. 图 11 所示, 随着钯在双金属中比例的增加, 甲醇的氧化峰电流值慢 慢增加. 这说明钯的加入能提高催化剂的催化活性. 但 是当钯的含量超过 $45 \%$ 后, 甲醇氧化峰电流值开始逐渐 下降，说明钯金属对于甲醇在酸性条件下氧化的催化活 性并不高. 该结果与以前的文献报道一致 ${ }^{[30 \sim 32]}$. 基于以 上实验结果，双金属对于甲醇氧化展现的高的催化活性

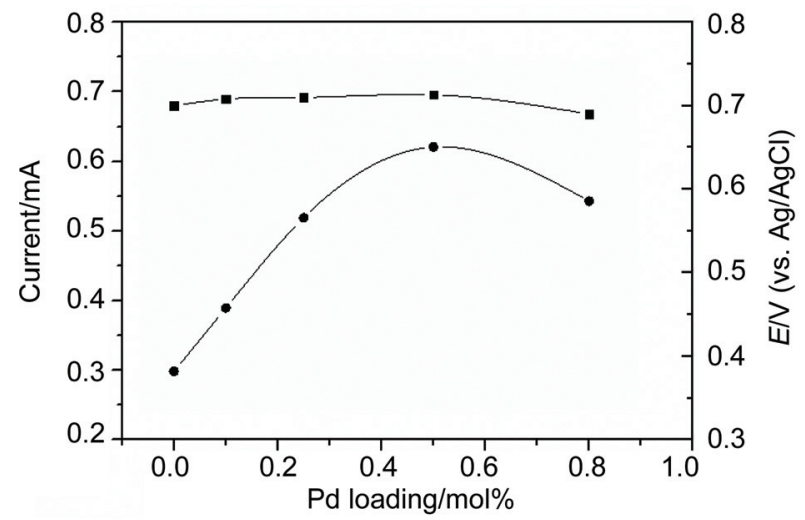

图 11 不同钯负载量的催化剂对于甲醇氧化性能的变化

Figure 11 The electrocatalytic performance of the as-prepared hybrids varied with the Pd loading

可解释为: 金属钯的加入在一定程度上增大了铂金属的 比表面积，使得更多的铂原子裸露在纳米粒子的表层， 从而产生较高的催化活性. 当钯的量进一步增大，高活 性的铂的比例必然降低, 进而导致催化剂活性的降低, 表现为峰电流变小. 综合考虑, 我们采取 45/55(钯/铂) 为最佳配比进行下一步实验.

计时电流技术是一个用来评估燃料电池催化剂性 能优劣的有用的方法 ${ }^{[33]}$. 3D PPG, PPG 和 E-TEK Pt/C 催 化剂典型的电流-时间曲线显示于图 12 中. 从图中可以 观察到: 在最初阶段, 三者都显示出相同的电流衰减趋 势，这与文献报道是一致的 ${ }^{[34]}$. 但是，随着时间的延长， 我们观察到 3D PPG 的电催化活性的损失小于 PPG 和 E-TEK Pt/C 催化剂, 从而说明 3D PPG 催化剂的稳定性 优于 PPG 和 E-TEK Pt/C 催化剂.

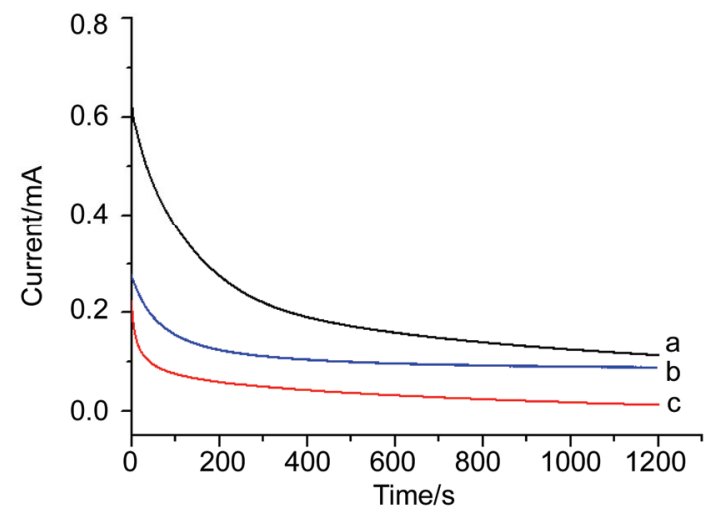

图 $123 \mathrm{D} \mathrm{PPG}, \mathrm{PPG}$ 和 E-TEK Pt/C 催化剂在 $0.5 \mathrm{~mol} / \mathrm{L} \mathrm{H}_{2} \mathrm{SO}_{4}+1$ $\mathrm{mol} / \mathrm{L} \mathrm{CH}_{3} \mathrm{OH}$ 溶液中电流密度-时间曲线, a: $3 \mathrm{DPPG}, \mathrm{b}$ : PPG, c: E-TEK $\mathrm{Pt} / \mathrm{C}$. 恒电位为 $0.7 \mathrm{~V}$, 其中催化剂质量为 $10^{-6} \mathrm{~g}$

Figure 12 The current-time curves of 3D PPG, PPG and E-TEK Pt/C in a $0.5 \mathrm{~mol} / \mathrm{L} \mathrm{H}_{2} \mathrm{SO}_{4}$ solution containing $1 \mathrm{~mol} / \mathrm{L}$ methanol. a: $3 \mathrm{D}$ PPG, b: PPG, c: E-TEK Pt/C. The potential was $0.7 \mathrm{~V}$, the mass of the electrocatalyst was $10^{-6} \mathrm{~g}$

\section{3 结论}

结合溶剂热方法和冰模板技术, 我们发展了一个简 
便地构建多孔铂钯双金属纳米粒子覆盖的石墨烯 (3D PPG)杂化体的新方法. 双金属纳米粒子的负载量可以 通过调节前驱物的比例进行有效地调控. 此外, 得到的 三维多孔杂化体能大大增强甲醇氧化的催化活性和稳 定性, 从而表明 $3 \mathrm{D}$ PPG 是一个高效的电催化剂. 我们 期待这个新的三维多孔杂化纳米材料能够在电催化剂, 传感器, 纳米电子学等领域发挥巨大的应用前景.

\section{4 实验部分}

GO 的制备: GO 依据 Hummers 方法合成 ${ }^{[35]}$.

PPG 杂化材料的制备: 向 $10 \mathrm{~mL}\left(1 \mathrm{mg} \cdot \mathrm{mL}^{-1}\right) \mathrm{GO}$ 的 $\mathrm{EG}$ 分散液中加入 $\mathrm{Na}_{2} \mathrm{PtCl}_{4}, \mathrm{Na}_{2} \mathrm{PdCl}_{4}$ (使二者物质的 量总和为 $0.05 \mathrm{mmol} / \mathrm{L})$ 和 $1.2 \mathrm{~g} \mathrm{PAA}$, 搅拌均匀. 然后 将混合物转移到 $15 \mathrm{~mL}$ 的聚四氟乙烯反应釜中, 使其在 $180{ }^{\circ} \mathrm{C}$ 下反应 $5 \mathrm{~h}$. 然后用去离子水清洗多次, 真空状 态下干燥备用.

3D PPG 结构组装: 将 PPG 配制成 $1 \mathrm{mg} \cdot \mathrm{mL}^{-1}$ 的水 分散液, 向其中加入 $1 \mathrm{~mL} \mathrm{3 \%}$ 的 PDDA 溶液, 超声 10 $\min$, 取 $100 \mu \mathrm{L}$ 上述混合液滴涂在玻碳片、玻璃片、硅 片等基底上; 室温下放置 $5 \mathrm{~min}$ 后, 将其转移到盛有液 氮的塑料容器中, 并保持 $2 \mathrm{~h}$. 待液氮挥发完毕后, 将基 底置于 $25{ }^{\circ} \mathrm{C}$ 的烘箱中干燥, 即可得到多孔的 3D PPG.

\section{References}

[1] Formo, E.; Lee, E.; CampbelL, D.; Xia, Y. N. Nano Lett. 2008, 8, 668.

[2] Sun, S. H.; Jaouen, F.; Dodelet, J. P. Adv. Mater. 2008, 20, 3900.

[3] Liang, H. P.; Zhang, H. M.; Hu, J. S.; Guo, Y. G.; Wan, L. J.; Bai, C. L. Angew. Chem., Int. Ed. 2004, 43, 1540.

[4] Wang, C.; Daimon, H.; Lee, Y.; Kim, J.; Sun, S. J. Am. Chem. Soc. 2007, 129, 6974.

[5] Tian, N.; Zhou, Z. Y.; Sun, S. G.; Ding, Y.; Wang, Z. L. Science 2007, 316, 732 .

[6] Yamauchi, Y.; Sugiyama, A.; Morimoto, R.; Takai, A.; Kuroda, K. Angew. Chem., Int. Ed. 2008, 47, 5371.

[7] Yamauchi, Y.; Takai, A.; Nagaura, T.; Inoue, S.; Kuroda, K. J. Am. Chem. Soc. 2008, 130, 5426.

[8] Wang, L.; Yamauchi, Y. J. Am. Chem. Soc. 2009, 131, 9152.
[9] Li, Y.; Gao, W.; Ci, L.; Wang, C.; Ajayan, P. M. Carbon 2010, 48, 1124.

[10] Huang, X.; Li, S. Z.; Huang, Y. Z.; Wu, S. X.; Zhou, X. Z.; Li, S. Z.; Gan, C. L.; Boey, F.; Mirkin, C. A.; Zhang, H. Nat. Commun. 2011, $2,292$.

[11] Kou, R.; Shao, Y.; Wang, D.; Engelhard, M. H.; Kwak, J. H.; Wang, J.; Viswanathan, V. V.; Wang, C.; Lin, Y.; Wang, Y.; Aksay, I. A.; Liu, J. Electrochem. Commun. 2009, 11, 954.

[12] Lu, J.; Do, I.; Drzal, L. T.; Worden, R. M.; Lee, I. ACS Nano 2008, 2,1825 .

[13] Guo, S.; Dong, S.; Wang, E. ACS Nano 2010, 4, 547.

[14] Tang, L. H.; Wang, Y.; Li, Y. M.; Feng, H. B.; Lu, J.; Li, J. H. Adv. Funct. Mater. 2009, 19, 2782.

[15] Zhou, M.; Zhai, Y. M.; Dong, S. J. Anal. Chem. 2009, 81, 5603.

[16] Bai, H.; Xu, Y.; Zhao, L.; Li, C.; Shi, G. Q. Chem. Commun. 2009, 1667.

[17] Jasuja, K.; Berry, V. ACS Nano 2009, 3, 2358.

[18] Guo, S. J.; Fang, Y. X.; Dong, S. J.; Wang, E. K. J. Phys. Chem. C 2007, 111, 17104.

[19] Guo, S. J.; Wang, L.; Dong, S. J.; Wang, E. K. J. Phys. Chem. C 2008, 112,13510

[20] Wang, S. Y.; Kristian, N.; Jiang, S. P.; Wang, X. Nanotechnology 2009, 20, 025605.

[21] Wang, S. Y.; Wang, X.; Jiang, S. P. Langmuir 2008, 24, 10505.

[22] Zhu, M. S.; Chen, P. L.; Liu, M. H. ACS Nano 2011, 5, 4529.

[23] Yoo, E.; Okata, T.; Akita, T.; Kohyyama, M.; Nakamura, J.; Honma, I. Nano Lett. 2009, 9, 2255.

[24] Estevez, L.; Kelarakis, A.; Gong, Q. M.; Da'as, E. H.; Giannelis, E. P. J. Am. Chem. Soc. 2011, 133, 6122.

[25] Deng, H.; Li, X.; Peng, Q.; Wang, X.; Chen, J.; Li, Y. Angew. Chem., Int. Ed. 2005, 44, 2782.

[26] Zheng, S. F.; Hu, J. S.; Zhong, L. S.; Wan, L. J.; Song, W. G. J. Phys. Chem. C 2007, 111, 11174.

[27] Wang, S. Y.; Jiang, S. P.; White, T. J.; Guo, J.; Wang, X. Adv. Funct. Mater. 2009, 19, 378.

[28] Stankovich, S.; Dikin, D. A.; Piner, R. D.; Kohlhaas, K. A.; Kleinhammes, A.; Jia, Y.; Wu, Y.; Nguyen, S. T.; Ruoff, R. S. Carbon 2007, 45, 1558.

[29] Sun, S. H.; Yang, D. Q.; Villers, D.; Zhang, G. X.; Sacher, E.; Dodelet, J. P. Adv. Mater. 2008, 20, 571.

[30] Casado-Rivera, E.; Volpe, D. L.; Alden, L.; Lind, C.; Downie, C.; Vazquez-Alvarez, T.; Angelo, A. C. D.; Disalvo, F. J.; Abruna, H. D. J. Am. Chem. Soc. 2004, 126, 4043.

[31] Mu, Y, Y.; Liang, H. P.; Hu, J.; Hu, J. S.; Jiang, L.; Wan, L. J. J. Phys. Chem. B 2005, 109, 22212.

[32] Zhang, H.; Yin, Y. J.; Hu, Y. J.; Li, C. Y.; Wu, P.; Wei, S. H.; Cai, C. X. J. Phys. Chem. C 2010, 114, 11861.

[33] Teng, X.; Liang, X.; Rahman, S.; Yang, H. Adv. Mater. 2005, 17 2237.

[34] Lin, Z. H.; Lin, M. H.; Chang, H. T. Chem. Eur. J. 2009, 15, 4656.

[35] Hummers, W. S.; Offeman, R. E. J. Am. Chem. Soc. 1958, 80, 1339. 